\title{
RENDIMIENTO Y CALIDAD DE GRANO EN LÍNEAS MEJORADAS DE FRIJOL CULTIVADAS BAJO RIEGO EN DURANGO, MÉXICO
}

\section{YIELD AND GRAIN QUALITY IN IMPROVED COMMON BEAN LINES GROWN UNDER IRRIGATION IN DURANGO, MEXICO}

\author{
Rigoberto Rosales-Serna', Hilario Flores-Gallardo'**, \\ Cynthia A. Nava-Berumen² e Ixchel A. Ortiz-Sánchez ${ }^{3}$
}

\begin{abstract}
IInstituto Nacional de Investigaciones Forestales, Agrícolas y Pecuarias, Campo Experimental Valle del Guadiana, Durango, México. ${ }^{2}$ Universidad Juárez del Estado de Durango, Programa Institucional de Doctorado en Ciencias Agropecuarias y Forestales, Durango, México. ${ }^{3}$ Instituto Tecnológico del Valle del Guadiana, Villa Montemorelos, Durango, México.
\end{abstract}

*Autor para correspondencia (flores.hilario@inifap.gob.mx)

\section{RESUMEN}

El estado de Durango sobresale en la generación de variedades de frijol (Phaseolus vulgaris L.) con alto impacto desde el punto de vista productivo y comercial en México. El presente estudio tuvo como objetivo evaluar 14 líneas mejoradas de frijol común con base en rendimiento, calidad de grano y atributos morfo-agronómicos. Los tipos de líneas fueron: pinto (7) negro opaco (4), alubia (2) y flor de mayo (1), las cuales se compararon con testigos comerciales. La siembra se realizó en Durango, México el 10 de julio de 2015, 07 de julio de 2016 y 21 de junio de 2017. Se utilizó un diseño completamente aleatorio (franjas apareadas), con parcela experimental de seis surcos de $25 \mathrm{~m}$ de largo y parcela útil de dos surcos de $5 \mathrm{~m}$ de longitud, $0.81 \mathrm{~m}$ de separación $\left(8.1 \mathrm{~m}^{2}\right)$ y cinco repeticiones. Se registraron los días a floración (DF), días a madurez fisiológica (DMF), rendimiento, peso de 100 semillas y se realizó análisis químico proximal del grano. Se aplicó análisis de varianza y la comparación de medias se realizó con la prueba de Tukey $(P \leq 0.05)$. Se observaron valores entre de 44 y 51 días después de siembra (dds) para DF y de entre 96 y 110 dds para DMF. Las líneas de frijol pinto mostraron precocidad a la floración (39 dds) y madurez (100 dds). Se obtuvieron diferencias significativas (de $P \leq 0.0001$ a $P \leq 0.04$ ) entre líneas para rendimiento de grano, con un promedio mayor en $2016\left(2673 \mathrm{~kg} \mathrm{ha}^{-1}\right)$ y variaciones para el tamaño del grano de 45.0 a $51.1 \mathrm{~g}$ por 100 semillas. No se detectaron diferencias estadísticamente significativas entre líneas para el contenido de cenizas ( 3.5 a $5.0 \%$ ), proteína cruda (20.5 a $25.1 \%$ ), grasa cruda (1.2 a $2.2 \%$ ), fibra cruda (1.9 a $4.4 \%$ ) y extracto libre de nitrógeno (65.8 a 71.2 $\%)$. Se identificaron líneas mejoradas sobresalientes de frijol con adaptación en riego, alto rendimiento y calidad nutricia y comercial del grano.

Palabras clave: Phaseolus vulgaris, clima, cruzamientos múltiples, rendimiento.

\section{SUMMARY}

The state of Durango stands out in the generation of common bean (Phaseolus vulgaris L.) varieties with high impact from the productive and commercial points of view in Mexico. The objective of this study was to evaluate 14 improved lines of common bean based on seed yield, grain quality and morpho-agronomic attributes. The types of lines were: pinto (7), opaqueblack (4), white (2) and pink (1), which were compared with commercial checks. The planting was carried out in Durango, Mexico on July $10^{\text {th }}, 2015$; July $07^{\text {th }}, 2016$ and June $21^{\text {th }}, 2017$. A completely randomized design (paired strips) was used, the experimental plot consisted of six rows of $25 \mathrm{~m}$ long and a useful plot of two rows of $5 \mathrm{~m}$ in length, $0.81 \mathrm{~m}$ separation $\left(8.1 \mathrm{~m}^{2}\right)$ and five replications. Days to flowering (DF) and to physiological maturity (DPM), yield and 100 -seeds weight were recorded, and a proximate chemical analysis of the grain was also performed. Analysis of variance was applied and the comparison of means was carried out with the Tukey test $(P \leq 0.05)$. Values were observed between 44 and 51 days after sowing (das) for DF and between 96 and 110 das for DPM. The pinto lines showed precocity at flowering (39 das) and maturity (100 das). Significant differences were obtained (from P $\leq$ 0.0001 to $P \leq 0.04$ ) between improved lines for seed yield, with higher average $\left(2673 \mathrm{~kg} \mathrm{ha}^{-1}\right)$ in 2016 and variation for seed size from 45.0 to $51.1 \mathrm{~g}$ per 100 seeds. No statistically significant differences were detected between lines for ash content (3.5 to $5.0 \%$ ), crude protein (20.5 to $25.1 \%$ ), crude fat (1.2 to $2.2 \%$ ), crude fiber (1.9 to $4.4 \%$ ) and nitrogen-free extract (65.8 to $71.2 \%$ ) Outstanding improved lines of common bean were identified with adaptation under irrigation, high yield and grain commercial quality.

Index words: Phaseolus vulgaris, weather, multiple crosses, yield

\section{INTRODUCCIÓN}

El estado de Durango sobresale en la generación de variedades de frijol (Phaseolus vulgaris L.) con alto impacto desde el punto de vista productivo y comercial en México. El programa de frijol y garbanzo (Cicer arietinum) del Instituto Nacional de Investigaciones Forestales, Agrícolas y Pecuarias (INIFAP) ha generado líneas mejoradas con diferentes tipos comerciales de grano y adaptación para condiciones de riego y temporal (Rosales et al., 2009). Dichas líneas mejoradas posibilitan el incremento de la productividad y competitividad del cultivo de frijol en Durango; sin embargo, es necesaria la validación del germoplasma mejorado en condiciones de campo (validación interna) y posteriormente en terrenos de productores cooperantes (validación externa), para seleccionar aquellas que cumplan con los requerimientos de los agricultores, comerciantes, industriales y consumidores. Los agricultores requieren variedades precoces, resistentes a enfermedades, con alto rendimiento, grano de fácil comercialización y precio alto El consumidor demanda un producto de calidad visual alta, tiempo de cocción reducido, pocos granos reventados durante la cocción y sabor agradable (Rosales-Serna et al., 2014). 
Actualmente existen en Durango avances considerables en el mejoramiento genético del frijol, ya que se cuenta con variedades y líneas con características deseables en precocidad, rendimiento y calidad comercial del grano, así como para tolerancia a algunos patógenos causantes de enfermedades como la antracnosis (Colletotrichum lindemuthianum) y roya (Uromyces appendiculatus var. appendiculatus) (Rosales et al., 2009). El germoplasma mejorado incluye líneas dentro de las clases comerciales de mayor importancia en México, con la finalidad de diversificar la producción y abastecer el déficit observado en el caso de grano pinto, flor de mayo, negro (opaco y brillante) y alubia (blanco); con ello se espera facilitar la comercialización del grano producido en Durango y mejorar el beneficio económico obtenido por los productores agrícolas dedicados al cultivo del frijol.

Los productores de frijol en Durango han manifestado la necesidad de incrementar el tamaño de la semilla en la variedad Pinto Saltillo debido a que el valor registrado en dicha variedad (<35 g por 100 semillas) reduce su aceptación en el mercado nacional e internacional; por ello, se han generado líneas y variedades mejoradas de frijol pinto con tamaño de grano mediano y grande (35 a $45 \mathrm{~g}$ por 100 semillas) (Rosales et al., 2015, Com. Pers.) ${ }^{1}$. También se han desarrollado líneas dentro de otras clases comerciales de grano con importancia económica en México, como es el caso de negro opaco (Jamapa), flor de mayo, alubia y azufrado. Dichas líneas carecen de validación en ambientes múltiples, lo cual limita su dominio de recomendación para siembras comerciales de frijol en Durango. Por lo anterior, el objetivo de la presente investigación fue evaluar un grupo de 14 líneas mejoradas de frijol, cultivadas bajo condiciones de riego en Durango, con base en rendimiento y calidad del grano, con miras a su uso comercial.

\section{MATERIALES Y MÉTODOS}

\section{Material genético}

Se sembró un grupo de 14 líneas mejoradas de frijol de diferente tipo comercial de grano: pinto (PT, 7), negro opaco $(N G O, 4)$, alubia $(A L, 2)$ y flor de mayo $(F M, 1)$. Las líneas provienen de cruzamientos biparentales dobles y múltiples obtenidos a partir de progenitores genéticamente distantes de las razas Durango (pinto), Jalisco (negro brillante y flor de mayo), Mesoamérica (negro opaco) y Nueva Granada (azufrado) (Cuadro 1).

\footnotetext{
${ }^{1}$ Rosales S. R., E. I. Cuéllar R. y L. M. Salazar S. (2015) Variedades para incrementar el rendimiento y la calidad del frijol. Campo Experimental Valle del Guadiana, INIFAP. Durango, Durango, México. 2 p.
}

\section{Sitio experimental}

El sitio experimental se localiza en las coordenadas geográficas $23^{\circ} 59^{\prime} 15^{\prime \prime}$ latitud N, 104 37' 17" longitud O y una altitud de $1879 \mathrm{~m}$. El suelo predominante es tipo francoarcilloso, con capacidad intermedia para la retención de humedad, profundidad media, pendiente de 0 a $2 \%$, pH 7.9 (moderadamente alcalino) y contenido pobre de materia orgánica, fósforo y nitrógeno. El clima predominante en la región es templado semiárido $\left[B S_{1} \mathrm{kw}(\mathrm{w})(\mathrm{e})\right]$, con régimen de lluvias en verano, variación fuerte de temperatura y media anual para dicha variable de $17.4^{\circ} \mathrm{C}$ (García, 1987). La lluvia acumulada durante el año alcanza un promedio de $476 \mathrm{~mm}$, con valores altos entre junio y septiembre (Medina et al., 2005). Durante el periodo de evaluación se registraron promedios para la temperatura máxima entre $26.8^{\circ} \mathrm{C}$ y $27.9^{\circ} \mathrm{C}$, mínima de 13.4 a $14.2^{\circ} \mathrm{C}$ y una precipitación acumulada entre 221 y 381 mm (Cuadro 2) (INIFAP, 2018).

\section{Diseño experimental y manejo en campo}

Se utilizó un diseño completamente aleatorio en franjas apareadas con cinco repeticiones. La parcela experimental consistió de seis surcos de $25 \mathrm{~m}$ de longitud y $0.81 \mathrm{~m}$ de separación y una parcela útil de dos surcos de $5 \mathrm{~m}$ de longitud y $0.81 \mathrm{~m}$ de separación $\left(8.1 \mathrm{~m}^{2}\right.$ ). La siembra se realizó durante tres ciclos agrícolas en primavera-verano (P-V): 10 de julio de 2015, 07 de julio de 2016 y 21 de junio de 2017 (Cuadro 2).

En todos los ciclos agrícolas de evaluación se realizaron dos escardas, una aplicación de herbicida [Flex® (Fomesafen) y Pivot ${ }^{\circledR}$ (Imazetapir), $0.25+1.0 \mathrm{~L} \mathrm{ha}^{-1}$ ], con la complementación de dos a tres deshierbes manuales para mantener el cultivo libre de maleza. La fertilización se aplicó durante la siembra y primera escarda, con base en la dosis recomendada en el paquete tecnológico emitido por el INIFAP para el frijol cultivado bajo condiciones de riego (35-50-00, $\mathrm{N}-\mathrm{P}_{2} \mathrm{O}_{5}-\mathrm{K}_{2} \mathrm{O}$ ). Se aplicaron entre uno y dos riegos de auxilio para evitar estrés hídrico severo de las plantas.

\section{Variables evaluadas}

Se registró el número de días a floración y a madurez fisiológica, contabilizados después de siembra (dds). En madurez fisiológica se tomaron cinco muestras obtenidas mediante un muestreo sistemático para determinar el rendimiento de grano $\left(\mathrm{kg} \mathrm{ha}^{-1}\right)$ y el peso de 100 semillas (g). Las muestras para la determinación del rendimiento se obtuvieron de dos surcos de $5 \mathrm{~m}$ de longitud con una separación de $0.81 \mathrm{~m}\left(8.1 \mathrm{~m}^{2}\right)$. 
Cuadro 1. Genealogía de líneas mejoradas de frijol utilizadas en pruebas de rendimiento y calidad del grano en Durango, México.

\begin{tabular}{ll}
\hline Línea & Genealogía \\
\hline PT14059 & OC/PTSaltillo-1 \\
PT14061 & OC/PTSaltillo-3 \\
PT14069 & PTSaltillo/PTColoso-2-1 \\
PT14036 & PTSaltillo/PTLibertad///PTClaro/PTSaltillo//PTSaltillo/PTVilla-2-17 \\
PT14029 & PTSaltillo/PTLibertad///PTClaro/PTSaltillo//PTSaltillo/PTVilla-2-10 \\
PT14055 & PTBayacora/Maverick///PTClaro/PTSaltillo//PTSaltillo/PTVilla-2-8 \\
PT14053 & PTBayacora/Maverick///PTClaro/PTSaltillo//PTSaltillo/PTVilla-2-6 \\
NG014013 & Navar174//AGS 466/AZREG87-1-19///Negro San Luis-6 \\
NG014063 & R.Gde/NGAltiplano-4-1//FNPNL-4 \\
NG014035 & Jamapa/NGAltiplano-5 \\
NG014014 & Navar174//AGS 466/AZREG87-1-19///Negro San Luis-7 \\
FM14011 & FMM38/FM 1/2 Oreja-11 \\
AL14008 & SPS-8 \\
AL Grande & SPS-1 \\
\hline PT: pinto,NG0: &
\end{tabular}

PT: pinto, NGO: negro opaco, FM: flor de mayo, AL: alubia.

Cuadro 2. Variables climáticas registradas en tres ciclos agrícolas P-V en Durango, México, en la evaluación de líneas mejoradas de frijol.

\begin{tabular}{lcccc}
\hline Ambiente & Fecha de Siembra & $\begin{array}{c}\text { Temperatura } \\
\text { Máxima }\left({ }^{\circ} \mathrm{C}\right)\end{array}$ & $\begin{array}{c}\text { Temperatura } \\
\text { Mínima }\left({ }^{\circ} \mathrm{C}\right)\end{array}$ & $\begin{array}{c}\text { Precipitación } \\
\text { Acumulada }(\mathrm{mm})\end{array}$ \\
\hline Durango, 2015 & $10 / 07 / 2015$ & 27.7 & 13.4 & 221 \\
Durango, 2016 & $07 / 07 / 2016$ & 26.8 & 14.2 & 357 \\
Durango, 2017 & $21 / 06 / 2017$ & 27.9 & 14.2 & 381 \\
\hline
\end{tabular}

\section{Análisis químico proximal}

Se realizó análisis químico proximal del grano cosechado en 2017, con base en las recomendaciones de la AOAC (1990). Se determinó el contenido de cenizas, grasa cruda, fibra cruda, proteína cruda y extracto libre de nitrógeno (ELN). La cantidad de cenizas se cuantificó mediante la incineración de los residuos de cada muestra a $550^{\circ} \mathrm{C}$. El contenido de grasa se determinó con un extractor Soxhlet (Soxtec Avanti 2055, Foss Tecator, Suecia) utilizando éter de petróleo como solvente (AOAC, 1990). La fibra cruda se determinó después de digerir las muestras, libres de grasa, en soluciones de ácido sulfúrico e hidróxido de sodio en orden sucesivo y luego se realizó la calcinación de los residuos (AOAC, 1990). El contenido de proteína cruda se midió con base en el método micro-Kjeldahl, tras multiplicar el valor de nitrógeno total por un factor de 6.25 (AOAC, 1990). El ELN (carbohidratos solubles) fue calculado mediante la sustracción, con respecto al 100 $\%$ de las fracciones de proteína cruda, cenizas, extracto etéreo y fibra cruda, expresadas con base en el peso seco.

\section{Análisis estadístico}

Se aplicó análisis de varianza para el rendimiento y peso de 100 semillas con el modelo de un diseño completamente aleatorio con cinco repeticiones y comparación de medias con la prueba de Tukey $(P \leq 0.05)$. En el caso del análisis químico proximal de grano se utilizó el modelo de un diseño completamente aleatorio con dos repeticiones. Los análisis se realizaron con el paquete estadístico SAS Ver. 9.4 (SAS Institute, 2013). 


\section{RESULTADOS Y DISCUSIÓN}

Durante el ciclo agrícola P-V 2015 se registró un promedio de 44 dds para floración y 105 dds para madurez fisiológica (Cuadro 3), mientras que durante el ciclo agrícola P-V 2016 la floración se presentó a los 44 dds y la madurez fisiológica a los 96 dds (Cuadro 4). En el ciclo P-V 2017, el promedio para la floración fue de 51 dds y de 110 dds para madurez fisiológica (Cuadro 5). Las variaciones observadas en la fenología se relacionaron con las condiciones ambientales (temperatura y precipitación acumulada) y con el tipo de germoplasma utilizado, debido a que durante 2017 se incluyeron testigos, que presentaron floración y madurez tardía (Flor de Mayo Media Oreja: floración 58 dds y madurez 125 dds) (Rosales et al., 2004).

En 2015 y en el grupo de líneas de grano pinto se registró precocidad a floración (PT14061 = 39 dds) y a madurez fisiológica (PT14036, PT14059 y PT14061 = 97 dds), en comparación con el resto del germoplasma evaluado. En 2016, el grupo de líneas de grano pinto mostró precocidad a floración (PT14059 = 40 dds y PT14061 = 41 dds) y a madurez fisiológica (PT14061 = 88 dds). En 2017, sobresalió por su precocidad a floración la línea PT14061 (46 dds) y a madurez fisiológica las líneas Negro 80 25, PT14029, PT14036 y PT14061 (100 dds). En todos los años sobresalió por su ciclo biológico corto el germoplasma de grano pinto, aunque se observaron otros materiales de grano negro opaco con precocidad a floración y madurez. A pesar de lo anterior, en 2017 la duración del ciclo biológico en las líneas de grano pinto registró el promedio mayor en comparación con otras variedades de frijol, como es el caso de Pinto Centauro (Rosales et al., 2015), la cual madura entre 81 dds y 97 dds. El incremento en el número de días a madurez se debió al uso de progenitores sensibles al fotoperiodo y con ciclo biológico de intermedio a tardío (Pinto Saltillo y Negro San Luis) (Rosales et al., 2004).

Se observaron diferencias significativas para el rendimiento entre las líneas evaluadas en 2015 ( $P \leq 0.0023)$, 2016 ( $P \leq 0.04)$ y 2017 ( $P \leq 0.0001$ ) (Cuadros 3, 4 y 5). El rendimiento promedio más alto se obtuvo en 2016, con $2673 \mathrm{~kg} \mathrm{ha}{ }^{-1}$ y fluctuaciones entre $1639 \mathrm{~kg} \mathrm{ha}^{-1}$ (AL14008) y $3508 \mathrm{~kg} \mathrm{ha}^{-1}$ (NG014014). El rendimiento observado en todas las líneas mejoradas durante 2016 se relacionó con la probable sobreestimación de esta variable debido al uso de progenitores genéticamente distantes (Darkwa et al., 2016; Da Silva et al., 2017), el ambiente favorable para el cultivo de frijol en siembras tempranas (junio) (Jiménez y Acosta, 2013) y el riego aplicado de forma oportuna (Rosales y Flores, 2017). En 2015, el rendimiento promedio fue de $1701 \mathrm{~kg} \mathrm{ha}^{-1}$ con valores entre 857 (NG014013) y $2653 \mathrm{~kg}$ ha $^{-1}$ (FM14011). En 2017, el rendimiento promedio fue de
$2319 \mathrm{~kg} \mathrm{ha}^{-1}$ con variaciones entre 561 (AL14008) y 3695 $\mathrm{kg} \mathrm{ha}^{-1}$ (NG014035). El rendimiento registrado en 2015 se debió principalmente a la cantidad baja de precipitación acumulada, lo cual en combinación con la temperatura y el fotoperiodo incrementaron la demanda atmosférica (Andriani, 2002) y con ello, se redujo la productividad del agua de riego (Rosales y Flores, 2017).

El rendimiento de grano registrado en las líneas evaluadas deberá corroborarse en siembras comerciales establecidas en Durango. Se observó que el uso de progenitores genéticamente distantes como Negro San Luis, México 332 y Pinto Claro, así como la obtención de cruzas múltiples favorecieron el vigor y rendimiento de las líneas obtenidas en ese ciclo de selección (Darkwa et al., 2016; Da Silva et al., 2017). Es importante mencionar que durante 2015 influyó negativamente la cantidad (221 $\mathrm{mm}$ ) y distribución de la precipitación acumulada, así como las variaciones en la temperatura, ya que a pesar de la aplicación de riego se tuvieron periodos de 5 a 30 días sin Iluvia considerable en varias ocasiones a lo largo del ciclo de cultivo, lo cual en combinación con las diferencias entre las temperaturas máximas y mínimas redujeron el rendimiento del frijol (Rosales et al., 2001; Rosales-Serna et al., 2004).

El tamaño de la semilla mostró variación a través de los ambientes de estudio; la línea mejorada AL14008 sobresalió en los tres años de prueba (Cuadros 3, 4 y 5) y superó al resto del germoplasma evaluado en 2015 con $45.2 \mathrm{~g}$ por 100 semillas, mientras que en 2016 presentó un valor de 45.0 g por 100 semillas y en 2017 la misma línea alcanzó $51.1 \mathrm{~g}$ por 100 semillas y con ello, la diferencia de peso con respecto a la variedad de mayor tamaño de la semilla, Bayo Victoria (54.4 g por 100 semillas) no fue estadísticamente significativa. Por su parte, el tamaño inferior de semilla se registró en la línea de grano negro opaco NG014063, con valores de $21.4 \mathrm{~g}$ por 100 semillas en $2015,22.9$ g por 100 semillas en 2016 y 28.1 g por 100 semillas en 2017, estadísticamente igual al valor obtenido por la variedad testigo Negro 8025 (25.5 g por 100 semillas). El tamaño de la semilla de esta última variedad cumplió parcialmente con los requisitos impuestos por el mercado de frijol negro opaco y pequeño (Jamapa), cuyo peso debe ser inferior a 25.0 g por 100 semillas (CIAT, 1987; Guzmán et al., 2009). Las líneas evaluadas mostraron valores distintos, y en algunos casos demasiado altos para los estándares comerciales (Guzmán et al., 2009), lo cual puede limitar su aceptación en el mercado.

No se detectaron diferencias significativas entre genotipos para la proporción de los compuestos nutrimentales contenidos en el grano (Cuadro 6). El contenido de cenizas (minerales) fluctuó entre 3.5 (Flor 
Cuadro 3. Características evaluadas en diferentes líneas mejoradas de frijol cultivadas en Durango, México, durante el ciclo agrícola P-V 2015.

\begin{tabular}{lcccc}
\hline Línea & Floración $(\mathrm{dds})$ & Madurez $(\mathrm{dds})$ & Rendimiento $\left(\mathrm{kg} \mathrm{ha}^{-1}\right)$ & Peso de 100 semillas $(\mathrm{g})$ \\
\hline PT14053 & 41 & 110 & $1544 \mathrm{abc}$ & 35.6 \\
PT14055 & 47 & 110 & $1403 \mathrm{abc}$ & 33.4 \\
PT14029 & 44 & 98 & $1993 \mathrm{abc}$ & 37.6 \\
PT14036 & 42 & 97 & $1490 \mathrm{abc}$ & 35.8 \\
PT14059 & 40 & 97 & $1762 \mathrm{abc}$ & 39.3 \\
PT14061 & 39 & 97 & $1535 \mathrm{abc}$ & 38.2 \\
PT14069 & 41 & 98 & $1831 \mathrm{abc}$ & 33.9 \\
NG014013 & 42 & 115 & $857 \mathrm{c}$ & 24.6 \\
NG014014 & 42 & 115 & $1233 \mathrm{bc}$ & 26.1 \\
NG014035 & 47 & $2033 \mathrm{abc}$ & 27.6 \\
NG014063 & 48 & $2343 \mathrm{ab}$ & 21.4 \\
FM14011 & 105 & $2653 \mathrm{a}$ & 34.0 \\
AL14008 & 45 & 105 & $1439 \mathrm{abc}$ & 45.2 \\
Alubia Grande & 43 & 104 & $1698 \mathrm{abc}$ & 42.7 \\
Promedio & 51 & 115 & 1701 & 34.0 \\
\hline
\end{tabular}

PT: pinto; NGO: negro opaco; FM: flor de mayo y AL: alubia. Medias con la misma letra no son estadísticamente diferentes (Tukey, P $\leq 0.05$ ).

Cuadro 4. Características evaluadas en diferentes líneas mejoradas de frijol cultivadas en Durango, México, durante el ciclo agrícola P-V 2016.

\begin{tabular}{lcccc}
\hline Línea & Floración $(\mathrm{dds})$ & Madurez $(\mathrm{dds})$ & Rendimiento $\left(\mathrm{kg} \mathrm{ha}^{-1}\right)$ & Peso de 100 semillas $(\mathrm{g})$ \\
\hline PT14053 & 43 & 98 & $3499 \mathrm{a}$ & 38.9 \\
PT14055 & 45 & 98 & $2987 \mathrm{a}$ & 37.5 \\
PT14029 & 42 & 91 & $2378 \mathrm{a}$ & 36.3 \\
PT14036 & 42 & 91 & $2604 \mathrm{a}$ & 37.0 \\
PT14059 & 40 & 90 & $3118 \mathrm{a}$ & 41.8 \\
PT14061 & 41 & 98 & $2234 \mathrm{a}$ & 38.4 \\
PT14069 & 42 & 94 & $2907 \mathrm{a}$ & 37.3 \\
NG014013 & 46 & 103 & $3031 \mathrm{a}$ & 31.8 \\
NG014014 & 47 & 104 & $3508 \mathrm{a}$ & 30.0 \\
NG014035 & 46 & $2628 \mathrm{a}$ & 26.5 \\
NG014063 & 47 & 99 & $2533 \mathrm{a}$ & 22.9 \\
FM14011 & 46 & $2507 \mathrm{a}$ & 35.6 \\
AL14008 & 43 & 94 & $1639 \mathrm{~b}$ & 45.0 \\
Alubia Grande & 50 & 98 & $1849 \mathrm{~b}$ & 42.1 \\
Promedio & 44 & 96 & 2673 & 35.8 \\
\hline
\end{tabular}

PT: pinto, NGO: negro opaco, FM: flor de mayo, AL: alubia. Medias con la misma letra no son estadísticamente diferentes (Tukey, P $\leq 0.05)$. 
Cuadro 5. Características evaluadas en diferentes líneas mejoradas de frijol cultivadas en Durango, México, durante el ciclo agrícola P-V 2017.

\begin{tabular}{|c|c|c|c|c|}
\hline Línea/Variedad & Floración (dds) & Madurez (dds) & Rendimiento (kg ha-1) & Peso de 100 semillas (g) \\
\hline NG014035 & 51 & 110 & 3695 a & $31.7 \mathrm{hi}$ \\
\hline Negro 8025 & 48 & 100 & $3357 a b$ & $25.5 \mathrm{k}$ \\
\hline NG014014 & 52 & 115 & $3281 \mathrm{abc}$ & $35.8 \mathrm{~g}$ \\
\hline РT14069 & 47 & 102 & 3159 abcd & 43.2 cde \\
\hline NG014013 & 51 & 118 & 3078 abcde & $34.7 \mathrm{gh}$ \\
\hline PT14053 & 52 & 110 & 2965 abcdef & 42.8 cde \\
\hline РT14055 & 52 & 110 & 2757 abcdef & 42.4 cdef \\
\hline FM14011 & 52 & 117 & 2731 bcdef & 42.0 def \\
\hline PT14029 & 49 & 100 & 2390 cdefgh & 43.2 cde \\
\hline РT14036 & 48 & 100 & 2362 cdefgh & $45.5 \mathrm{c}$ \\
\hline РT14061 & 46 & 100 & 2238 defghi & $49.0 \mathrm{~b}$ \\
\hline Pinto Saltillo & 48 & 102 & 2185 efghi & 40.7 ef \\
\hline FM Media Oreja & 58 & 125 & 2091 fghi & $39.3 \mathrm{f}$ \\
\hline Negro Verdín & 48 & 105 & 2037 ghi & $29.2 \mathrm{ij}$ \\
\hline NG014063 & 54 & 120 & 1951 ghi & $28.1 \mathrm{jk}$ \\
\hline РT14059 & 47 & 102 & 1834 ghi & $49.1 \mathrm{~b}$ \\
\hline Negro San Luis & 52 & 120 & 1723 hi & $32.6 \mathrm{gh}$ \\
\hline Bayo Victoria & 51 & 110 & 1406 ij & $54.4 \mathrm{a}$ \\
\hline Alubia Grande & 56 & 120 & $571 \mathrm{j}$ & $44.6 \mathrm{~cd}$ \\
\hline AL 14008 & 52 & 107 & $561 j$ & $51.1 \mathrm{ab}$ \\
\hline Promedio & 51 & 110 & 2319 & 40.0 \\
\hline
\end{tabular}

NGO: negro opaco, PT: pinto, FM: flor de mayo, AL: alubia. Medias con la misma letra en las columnas no son estadísticamente diferentes (Tukey, P $\leq 0.05)$.

de Mayo Media Oreja) y 5.0 \% (PT14069 y Negro Verdín); proteína cruda de 20.5 (NG014025) a $25.1 \%$ (PT14059); grasa cruda entre 1.2 (PT14059 y PT14061) y $2.2 \%$ (NG014035); fibra cruda de 1.9 (NG014013) a $4.4 \%$ (FM14011) y ELN entre 65.8 (FM14011) y $71.2 \%$ (PT14061). La dificultad para detectar diferencias estadísticas entre líneas y variedades se relacionó con la variabilidad de los resultados obtenidos, derivado del análisis del grano obtenido de las repeticiones de campo; además, influyó la interacción genético-ambiental y las variaciones ocasionadas por la segregación intrapoblacional derivadas del uso de cruzamientos dobles y múltiples, por lo que es recomendable incrementar el número de repeticiones.

Los análisis químicos proximales mostraron resultados similares a los obtenidos en otros estudios realizados en grano de frijol, en los cuales se obtuvieron valores para el contenido de cenizas de entre 3.8 y $4.4 \%$, proteína de entre 20.7 y $26.0 \%$ y grasa de entre 0.93 y $1.64 \%$ (MuñozVelázquez et al., 2009; Fernández y Sánchez, 2017). Aunque los valores fueron altos en relación con los obtenidos en el presente estudio para fibra cruda, con niveles de 5.55 a 19.86 \% y bajos para ELN con 38.07 a 60.09 \% (Fernández y Sánchez, 2017). Los valores observados en el germoplasma mejorado resultaron similares a los niveles presentes en las variedades cultivadas actualmente en Durango (Pinto Saltillo y Negro San Luis), las cuales fueron utilizadas como testigos comerciales. Con lo anterior, puede afirmarse que se mantuvieron las propiedades nutricias del frijol en las líneas mejoradas y, en algunos casos, sería posible obtener mayor nivel de rendimiento y calidad de grano. 
Cuadro 6. Resultados del análisis químico proximal en diferentes líneas mejoradas de frijol cultivadas en Durango, México durante el ciclo agrícola P-V 2017.

\begin{tabular}{lccccc}
\hline Línea/Variedad & Cenizas (\%) & Proteína Cruda (\%) & Grasa Cruda (\%) & Fibra Cruda (\%) & $\begin{array}{c}\text { Extracto Libre de } \\
\text { Nitrógeno (\%) }\end{array}$ \\
\hline PT14036 & 4.7 & 22.8 & 1.8 & 2.4 & 68.2 \\
PT14055 & 3.9 & 22.6 & 1.7 & 3.2 & 68.6 \\
PT14069 & 5.0 & 24.5 & 1.9 & 2.4 & 66.2 \\
PT14053 & 4.7 & 22.3 & 1.4 & 2.9 & 68.8 \\
PT14059 & 4.8 & 25.1 & 1.2 & 2.5 & 66.3 \\
PT14029 & 4.5 & 23.1 & 1.4 & 2.7 & 68.3 \\
PT14061 & 3.9 & 21.7 & 1.2 & 2.0 & 71.2 \\
Pinto Saltillo & 4.1 & 22.4 & 1.6 & 2.5 & 69.5 \\
NG014014 & 4.8 & 21.8 & 1.3 & 2.5 & 69.6 \\
NG014035 & 4.7 & 22.0 & 2.2 & 2.9 & 68.3 \\
NG014025 & 4.8 & 20.5 & 1.9 & 2.9 & 69.8 \\
Negro Verdín & 5.0 & 21.5 & 1.8 & 3.0 & 68.7 \\
NG014063 & 4.9 & 24.8 & 1.5 & 2.8 & 66.0 \\
NG014013 & 4.9 & 23.6 & 1.7 & 1.9 & 67.8 \\
Negro San Luis & 4.6 & 22.6 & 1.9 & 2.4 & 68.5 \\
FM14011 & 4.1 & 23.9 & 1.9 & 4.4 & 65.8 \\
Flor de Mayo Media Oreja & 3.5 & 21.9 & 1.6 & 2.2 & 70.9 \\
Bayo Victoria & 4.2 & 20.8 & 2.0 & 3.1 & 69.8 \\
AL14008 & 4.2 & 22.8 & 1.7 & 2.0 & 69.3 \\
Alubia Grande & 4.4 & 24.7 & 1.6 & 2.3 & 67.0 \\
Promedio & 4.5 & 22.8 & 1.7 & 2.6 & 68.4 \\
\hline PT:P & & & & \\
\hline
\end{tabular}

PT: pinto, NGO: negro opaco, FM: flor de mayo, AL: alubia.

\section{CONCLUSIONES}

Se identificaron dos líneas mejoradas de frijol sobresalientes en Durango (PT14069 y NG014035), con base en su rendimiento, calidad comercial del grano y adaptación en condiciones de riego. Se mantuvieron las características nutricias del grano, ya que todas las líneas mostraron valores similares a los registrados por las variedades usadas actualmente en siembras comerciales de frijol en Durango. El uso de padres genéticamente distantes y cruzamientos múltiples favoreció el incremento del rendimiento potencial obtenido con líneas mejoradas de frijol en Durango, lo cual debe complementarse con la siembra en ambientes favorables para el cultivo y riego aplicado oportunamente. Es recomendable que las líneas sobresalientes sean validadas en parcelas comerciales para verificar sus niveles de rendimiento y calidad del grano.

\section{AGRADECIMIENTOS}

La presente publicación formó parte del proyecto "Opciones tecnológicas para el desarrollo integral y sostenible de la producción agropecuaria y forestal en Durango", financiado con fondos fiscales INIFAP (Clave SIGI: 9573834038).

\section{BIBLIOGRAFÍA}

Andriani J. M. (2002) Estrés hídrico en soja. Idia XXI 2:48-51

AOAC, Association of Official Analytical Chemists (1990) Official Methods of Analysis. $15^{\text {th }}$ edition. Association of Official Analytical Chemists. Arlington, VA, USA. 1285 p.

CIAT, Centro Internacional de Agricultura Tropical (1987) Sistema Estándar para la Evaluación de Germoplasma de Frijol. A. van Schoonhoven y M. A. Pastor-Corrales (comps.). Centro Internacional de Agricultura Tropical. Cali, Colombia. 56 p.

Darkwa K., D. Ambachew, H. Mohammed, A. Asfaw and M. W. Blair (2016) Evaluation of common bean (Phaseolus vulgaris L.) genotypes for drought stress adaptation in Ethiopia. The Crop Journal 4:367-376, https://doi.org/10.1016/j.cj.2016.06.007 
Da Silva F. A., A. M. Corrêa, P. E. Teodoro, K. V. Lopes and C. C. G. Corrêa (2017) Genetic divergence in the common bean (Phaseolus vulgaris L.) in the Cerrado-Pantanal ecotone. Genetics and Molecular Research 16:gmr16019570, https://doi.org/10.4238/ gmr16019570

Fernández V. A. F. y E. Sánchez C. (2017) Estudio de las propiedades fisicoquímicas y calidad nutricional en distintas variedades de frijol consumidas en México. Nova Scientia 9:133-148.

García E. (1987) Modificaciones al Sistema de Clasificación Climática de Köppen (Para Adaptarlo a las Condiciones de la República Mexicana). $4^{a}$ edición. Instituto de Geografía, UNAM. México, D. F. 91 p.

Guzmán T. I., E. Almanza A., J. A. Acosta G. y S. H. Guzmán M. (2009) Estudio comparativo de características de calidad entre genotipos de frijol de grano negro. Agricultura Técnica en México 35:449-457.

INIFAP, Instituto Nacional de Investigaciones Forestales, Agrícolas y Pecuarias (2018) Red nacional de estaciones agrometeorológicas automatizadas INIFAP. Datos y recursos. INIFAP. Pabellón de Arteaga, Aguascalientes, México. http://clima.inifap.gob.mx/ Inmysr/Estaciones/MapaEstaciones. (Abril 2018).

Jiménez G. J. C. y J. A. Acosta G. (2013) Efecto y correlación de fechas de siembra, fertilización y densidad en el rendimiento de frijol Pinto Saltillo de temporal en Chihuahua. Revista Mexicana de Ciencias Agrícolas 4:115-127.

Medina G. G., G. Díaz P., J. López H., J. A. Ruiz C. y M. Marín S. (2005) Estadísticas Climatológicas Básicas del Estado de Durango (Periodo 1961-2003). Libro Técnico Núm. 1. Campo Experimental Valle del Guadiana, INIFAP. Durango, Durango, México. 224 p.

Muñoz-Velázquez E. E., D. Rubio-Hernández, I. Bernal-Lugo, R. Garza-García y C. Jacinto-Hernández (2009) Caracterización de genotipos nativos de frijol del estado de Hidalgo, con base a calidad del grano. Agricultura Técnica en México 35:429-438.

Rosales S. R., R. Ochoa M. y J. A. Acosta G. (2001) Fenología y rendimiento del frijol en el altiplano de México y su respuesta al fotoperiodo. Agrociencia 35:513-523

Rosales S. R., J. A. Acosta G., F. J. Ibarra P., E. I. Cuéllar R. y C. A. Nava B. (2009) Validación de variedades y líneas mejoradas de frijol en Durango. Publicación Especial Núm. 27. INIFAP-Campo Experimental Valle del Guadiana. Durango, Durango, México. $92 \mathrm{p}$.

Rosales S. R., J. A. Acosta G., J. S. Muruaga M., J. M. Hernández C., G. Esquivel E. y P. Pérez H. (2004) Variedades mejoradas de frijol del Instituto Nacional de Investigaciones Forestales Agrícolas y Pecuarias. Libro Técnico 6. Campo Experimental Valle de México, INIFAP. Chapingo, Estado de México. 148 p.

Rosales S. R. y H. Flores G. (2017) Importancia del agua de riego para la producción sostenible de frijol en Durango. Folleto Técnico Núm. 91. Campo Experimental Valle del Guadiana INIFAP. Durango, Durango, México. 28 p.

Rosales-Serna R., J. Kohashi-Shibata, J. A. Acosta-Gallegos, C. Trejo-López, J. Ortiz-Cereceres and J. D. Kelly (2004) Biomass distribution, maturity acceleration and yield in drought-stressed common bean cultivars. Field Crops Research 85:203-211, https://doi. org/10.1016/S0378-4290(03)00161-8

Rosales-Serna R., C. A. Nava-Berumen, H. González-Ramírez, M. D. Herrera, J. C. Jiménez-Galindo, N. Y. Z. Ramírez-Cabral y E. S. Osuna-Ceja (2014) Rendimiento, preferencia y calidad de enlatado de variedades de frijol pinto producidas en Durango, México. Revista Mexicana de Ciencias Agrícolas 5:309-315.

SAS Institute (2013) Base SAS® 9.4 Procedures Guide: Statistical Procedures. Second edition. SAS Institute Inc. Cary, NC, USA. $550 \mathrm{p}$. 